\title{
ON THE GENUS OF THE IDEMPOTENT GRAPH OF A FINITE COMMUTATIVE RING
}

\author{
G. Gold Belsi ${ }^{1}$, S. Kavitha ${ }^{2}$ \\ AND \\ K. Selvakumar ${ }^{1}$ \\ Reg. No. 18114012092031 \\ ${ }^{1}$ Department of Mathematics \\ Manonmaniam Sundaranar University \\ Tirunelveli 627 012, Tamil Nadu, India \\ ${ }^{2}$ Department of Mathematics \\ Gobi Arts and Science College \\ Gobichettipalayam 638 476, Tamilnadu \\ e-mail: goldbelsi@gmail.com \\ kavithaashmi@gmail.com \\ selva_158@yahoo.co.in
}

\begin{abstract}
Let $R$ be a finite commutative ring with identity. The idempotent graph of $R$ is the simple undirected graph $I(R)$ with vertex set, the set of all nontrivial idempotents of $R$ and two distinct vertices $x$ and $y$ are adjacent if and only if $x y=0$. In this paper, we have determined all isomorphism classes of finite commutative rings with identity whose $I(R)$ has genus one or two. Also we have determined all isomorphism classes of finite commutative rings with identity whose $I(R)$ has crosscap one. Also we study the the book embedding of toroidal idempotent graphs and classify finite commutative rings whose $I(R)$ is a ring graph.
\end{abstract}

Keywords: idempotent graph, planar, genus, crosscap.

2010 Mathematics Subject Classification: 13A15, 13M05, 05C25, 05C75.

\section{REFERENCES}

[1] S. Akbari, M. Habibi, A. Majidinya and R. Manaviyat, On the Idempotent Graph of a Ring, J. Algebra 12 (2013) 1-14. doi:10.1142/S0219498813500035 
[2] M.F. Atiyah and I.G. Macdonald, Introduction to Commutative Algebra (Addison - Wiley Publishing Company, London, 1969).

[3] I. Beck, Coloring of commutative rings, J. Algebra 116 (1988) 208-226. doi:10.1016/0021-8693(88)90202-5

[4] J.A. Bondy and U.S.R. Murty, Graph Theory and its Applications (American Elsevier, New York, 1976).

[5] F.R. Bernhart and P.C. Kainen, The book thickness of a graph, J. Comb. Theory, Ser. B 27 (1979) 320-331. doi:10.1016/0095-8956(79)90021-2

[6] S. Kavitha and R. Kala, On the genus of graphs from commutative rings, AKCE Int. J. Graphs Combin. 14 (2017) 27-34. doi:10.1016/j.akcej.2016.11.006

[7] T.Y. Lam, A First Course in Non-commutative Rings (Springer-Verlag, New York, 2001).

[8] M.M. Sysło, Characterizations of Outer planar graphs, Discrete Math. 26 (1979) $47-53$. doi:10.1016/0012-365X(79)90060-8

[9] A. Mallika and R. Kala, Nilpotent graphs with crosscap at most two, AKCE Int. J. Graphs Combin. 15 (2018) 229-237. doi:10.1016/j.akcej.2017.11.006

[10] M. Yannakakis, Embedding planar graphs in four pages, J. Comput. Syst. Sci. 38 (1989) 36-67. doi:10.1016/0022-0000(89)90032-9

[11] R. Blankenship and B. Oporowski, Drawing subdivisions of complete and complete bipartite graphs on books (Tech Rep. 1994-4, Department of Mathematics, Louisiana State University, 1999).

[12] T. McKenzie and S. Overbay, Book thickness of toroidal zero-divisor graphs, Africa Matematika 28 (2017) 823-830. doi:10.35834/mjms/1449161362

[13] T. Endo, The page number of toroidal graph is at most seven, Discrete Math. 175 (1997) 87-96. doi:10.1016/S0012-365X(96)00144-6

[14] A.T. White, Graphs, Groups and Surfaces (North-Holland-Amsterdam, 1984).

Received 9 March 2020 Revised 16 March 2020 Accepted 23 April 2020 